\title{
Denoising of MST RADAR Signal usingCWT and Overlapping Group Shrinkage
}

\author{
${ }^{1}$ P. Suresh Babu, ${ }^{2}$ Dr. G. Sreenivasulu \\ ${ }^{1}$ Associate. Professor, Department of ECE, S V Engineering College, Tirupati \\ ${ }^{2}$ Professor, Department of ECE, S V U College of Engineering, SVU, Tirupati
}

Article History: Received: 11 January 2021; Accepted: 27 February 2021; Published online: 5 April 2021

\begin{abstract}
Existing algorithmsare generally denouncing the existence of clusters with large amplitude coefficients. The L1 norm as well as other distinct models of sparsity does not attract a cluster tendency (group sparsity). In the light of a minimisation of convex cost work fusing the blended norm, this work introduces the technique "overlapping group shrinking." The groups are completely overlapping in order to abstain from blocking relics. A basic minimization calculation, in light of progressive replacement, is inferred. A straightforward strategy for setting the regularization boundary, in view of constricting the noise to a predefined level, is portrayed in detail by combining OGS with one of the most powerful mathematical tool wavelet transforms. In fact, the CWT coefficients are processed by OGS to produce a noise-free signal. The CWT coefficients are also processed.The proposed approach is represented on MST RADAR signals, the denoised signals delivered by CWT combined with OGS are liberated from noise.
\end{abstract}

Keywords: Overlapping Group Shrinkage (OGS), Denoising, DT-CWT, MST Radar data, Custom Thresholding, Improved Thresholding.

\section{INTRODUCTION}

Discrete Wavelet Transformation (DWT) seems to be a compelling arithmetic tool capable of solving many problems in several fields. As a powerful tool at its time, DWT had also been intrigued by most and many others who worked with applications and by a more comprehensive class of signal processing society, who identified that DWT showed some degree of confusion for instance with complex or modelled signals (for example Radar, Speech, and Music). In this respect, the CWT will produce substantial changes in the execution over the DWT. The Double-Tree-Complete Wavelet Transformation (CWT) was initiated by Kingsbury in 1998 for the first time in moderately continuous upgrading, $[1,2]$ it is roughly shifting invariant and permits a 2 nd and higher dimension of the directional wavelet with only $2 x 1 D$ Redundancies [3]. It is used particularly in signal processing systems for many scientific research papers. With substantial extra characteristics: it is often virtually invariantly moving, Directionally in two and higher sizes. It would have an account of higher of only $2 \mathrm{~d}$, generously less than the undecided DWT for d dimension functions. The CWT double (M-D) tree is non-separable but is based on a reliable Filter bank (FB).

The algorithm Wang et al. [4] has grown with NeighCoeff's shrinkage, namely DTCWT. Wang et al. 's work [4] found that the vibration signals DTCWT denoising approach is successful as opposed to NeighCoeff shrinking denotation methods based on DWT and SGWT. DTCWT decreases the vibration aliasing rate and is almost invariant in transitions, with desirable characteristics that are conducive to both tracking, and machinery diagnosis. For Doppler approximation and wind speed estimation of RADAR signals from the Indian MST RADAR at the National Atmospheric Research Laboratory, Gadanki P. Suresh Babu et al [5] developed the over-lap group denoising algorithm for estimating doppler speed. This work of denoising radar signals proves much betterwith DWT itself. With the promising results from work of P. Suresh Babu et al [5], is considered as the base in this study and owing to theadvantages of DTCWT, a method incorporating DTCWT with Overlapping GroupShrinkage (OGS) is employed to denoiseMST RADAR signals this work.

\section{COMPLEX WAVELET TRANSFORM}

Complex wavelet transformations use complicated filtration that disrupt real and imagined signals into the transformation domain. Real and imaginary coefficients were used to measure information about magnitude and phase, a kind of information needed in order to correctly describe the position of the energy of swelling functions. The Fourier transformtion is depend on a wide range sinusoidal oscillation.

$$
e^{j \Omega t}=\cos (\Omega t)+j \sin (\Omega t) \mathrm{e}
$$

The appropriate complex-valued wavelet and complex scaling function are provided as

$$
\psi_{c}(t)=\psi_{r}(t)+j \psi_{i}(t)
$$


In which $\psi_{r}(t)$ is even and real,

$j \psi_{i}(t)$ will beodd and imaginary.

In [6], through defining a complex extension of areal signal $f(t)$, Gabor presented Hilbert in and out of signal theory:

$$
x(t)=f(t)+j g(t)
$$

whereas, $g(t)$ represents the Hilbert transform of $f(t)$ and specified as $H\{f(t)\}$ and $j=(-1)^{1 / 2}$.

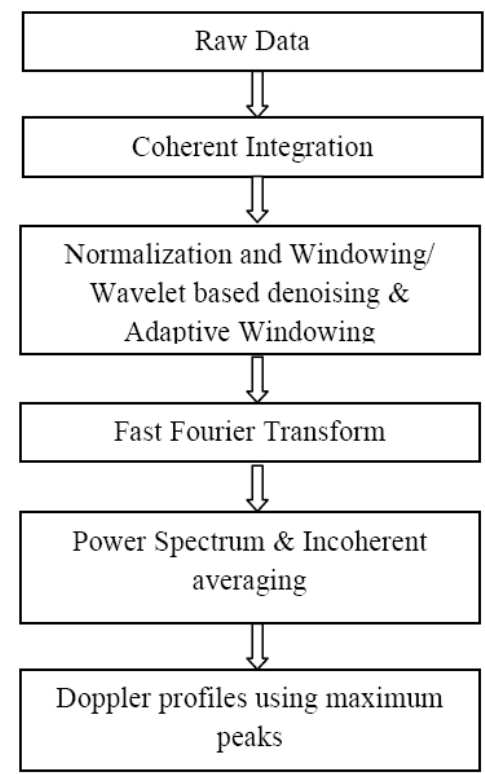

Fig1.Existing AlgorithmFlow chart

As seen in the diagram, the signals $\mathrm{g}(\mathrm{t})$ are the version $90^{\circ}$ shifted of $\mathrm{f}(\mathrm{t})$. The true part $\mathrm{f}(\mathrm{t})$ and the imaginary part $\mathrm{g}(\mathrm{t})$ of the analytical signal $\mathrm{x}(\mathrm{t})$ is also referred to in Hilbert as the 'Hardy Space.' The orthogonal symbol $\mathrm{g}(\mathrm{t})$ is $\mathrm{f}(\mathrm{t}) . \mathrm{G}(\mathrm{t})$ can be shown as [7] in the time domain.

$$
g(t)=H\{f(t)\}=\frac{1}{\pi} \int_{-\infty}^{\infty} \frac{f(t)}{t-\tau} d \tau=f(t) * \frac{1}{\pi t}
$$

Consider $F(\omega)$ as the Fourier transformation of signal $f(t) \& G(\omega)$ as the Fourier transformation of signal $g(t)$, the Hilbert transform relationship in the frequency domain between $\mathrm{f}(\mathrm{t})$ and $\mathrm{g}(\mathrm{t})$ is then provided by

$$
G(\omega)=F\{H\{f(t)\}\}=-j \operatorname{sgn}(\omega) F(\omega)
$$

where as, $-j \operatorname{sgn}(\omega)$ will be a deviated 'signum' function.

This analytical extension provides the approximate instantaneous frequency and amplitude of the $\mathrm{X}(\mathrm{t})$ signal:

Magnitude is given $x(t)=\sqrt{f^{2}(t)+g^{2}(t)}$

Angle is given $x(t)=\tan ^{-1}\{g(t) / f(t)\}$

The nonnegative spectral depiction in the Fourier fields[7] and[8], are another particular advantage of this quadrant, that further contributes to half the use of bandwidth. Reduced bandwidth usage is useful, particularly in multiple signal processing applications, to prevent aliasing philtre bands. The reduced philtre strip aliasing is the key to CWT 's shift invariant property. The so-called dual-transform wavelet transforms in one dimension represent an $\mathrm{x}(\mathrm{n})$ signal in terms of complex wavelets, consisting of actual and imaginary components that are in turn wavelets. Fig 2 demonstrates three steps of wavelet transformation Analysis and Synthesis of the Dual Tree Complex.

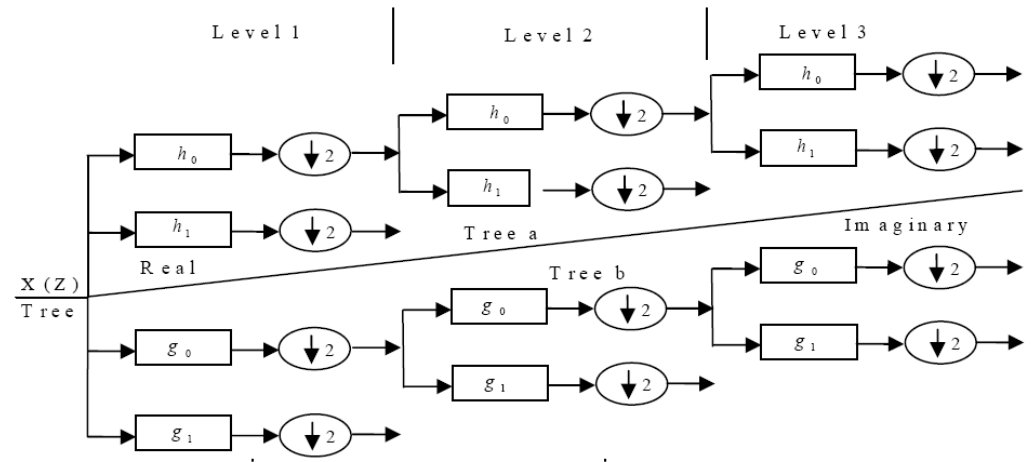




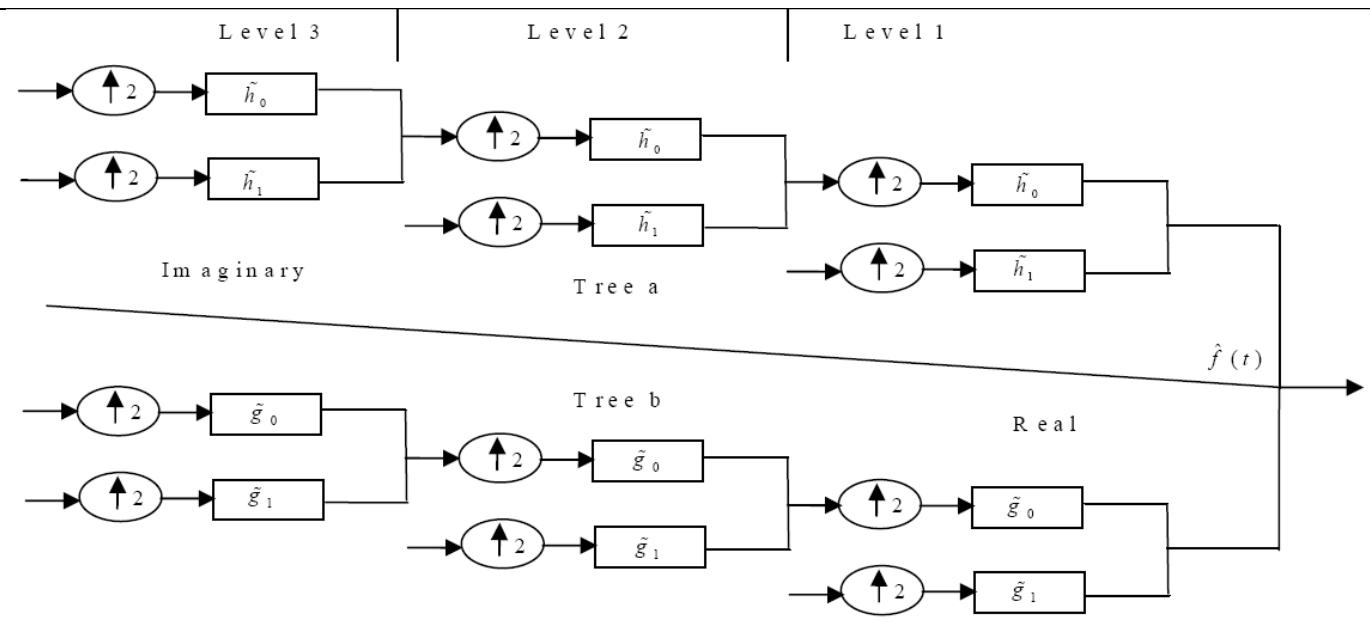

Fig2. Analysis \& Synthesis Filter banks for 3 stages

\section{PROPOSED ALGORITHM}

The RADAR signal cleaning algorithm is as follows with CWT with a group shrink.

1) Consider $f(n)$ as the RADAR signal, for $\mathrm{n}=0,1, \ldots, \mathrm{N}-1$.

2) Start generating noisy signal $\mathrm{x}(n)$ utilizing $x(n)=f(n)+\sigma z(n)$, where $\sigma$ standard deviation of noise

3) The filtration $\mathrm{h} 0, \mathrm{~h} 1$ and the other tree using philtres $\mathrm{g} 0 . \mathrm{g} 1$ input $\mathrm{y}(\mathrm{n})$ on both tree DWT trees using one tree.

4) In the two trees, apply OGS to wavelet transforms.

5) Using those same wavelet threshold coefficients, determine IDWT.

6) In order to produce the initial measure, the coefficients of both trees are then weighted. There after variance is accumulated by $\left(y(n)-\sum_{n=0}^{N-1} y(n)\right)^{2}$, where $\mathrm{y}(\mathrm{n})$ is the denoised signal.

\section{RESULTS \& DISCUSSION}

The below portion presents the results of various experiments of the specified method, as existing method shows better results with DWT based OGS, here directly, the RADAR signal is processed with CWT based OGS. As one can observe from the following results that complex wavelet transform based overlapping group shrinkage is producing better results, when compared with the existing DWT based OGS method. The following figure shows original and denoised spectrum of MST RADAR signal taken on 02 July 2015 from Indian MST RADAR, NARL situated at Gadanki.
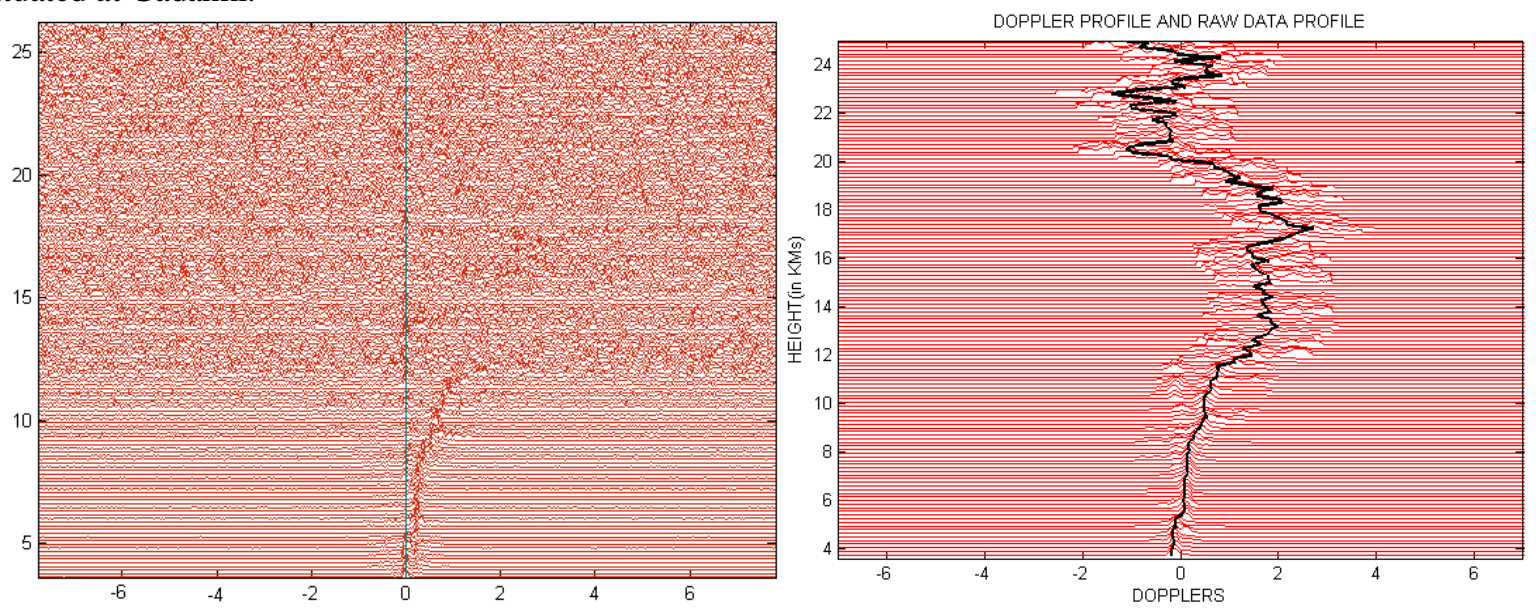

Fig3. Typical Spectra of Typical spectra of the east beam (a) Original (b) Denoised spectrum with adaptive window.

The distribution of theatmospheric content is shown in Fig after rendering adaptive denouncements for the eastern beam only. 3(b).From Fig. 3(b), it is clear that in the presence of atmospheric noise the Doppler can be obtained in higher altitudes. In order not to apply single derox for the full frame of data, bin wise denoising would reduce the likelihood of filtering the actual peak and increase the detectability of the signal.

Figure 4 shows the component of wind speed computed with DWT based OGS, CWT-based OGS and GPS[9] probe. From the figure. 4 it is clear that, over $15 \mathrm{~km}$, Doppler profiles are not accurately detected by the existing method; 


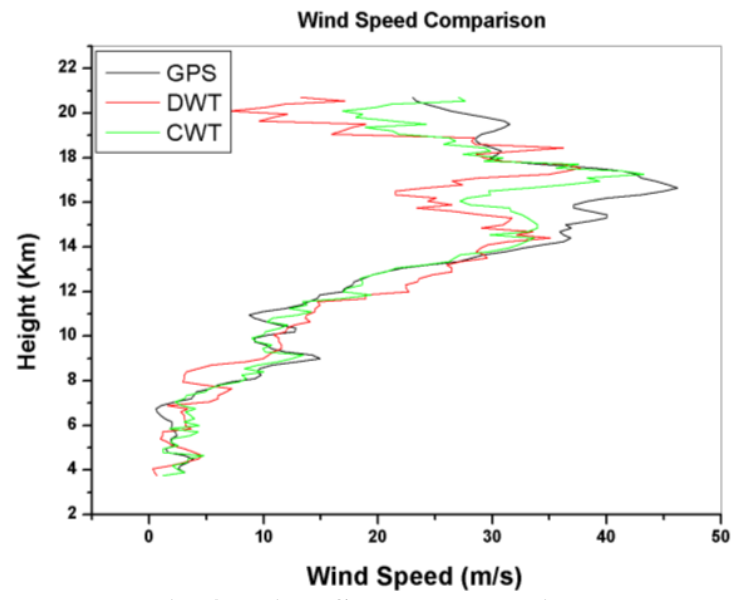

Fig 4. Wind Speed comparison

An exceedingly limited departure with the PALG is detected. Perhaps a small deviation is attributable, that the data utilized at the PALG has been collected vertically either from reflected echoes in the atmosphere's layers, without drifting in the horizontal direction, whereas horizontal drift of the globes can be achieved by high wind speeds among the collected data by the GPS. The proposed method using the OGS denotes adaptive window denotation method based on CWT could be seen to produce independent height profiles.

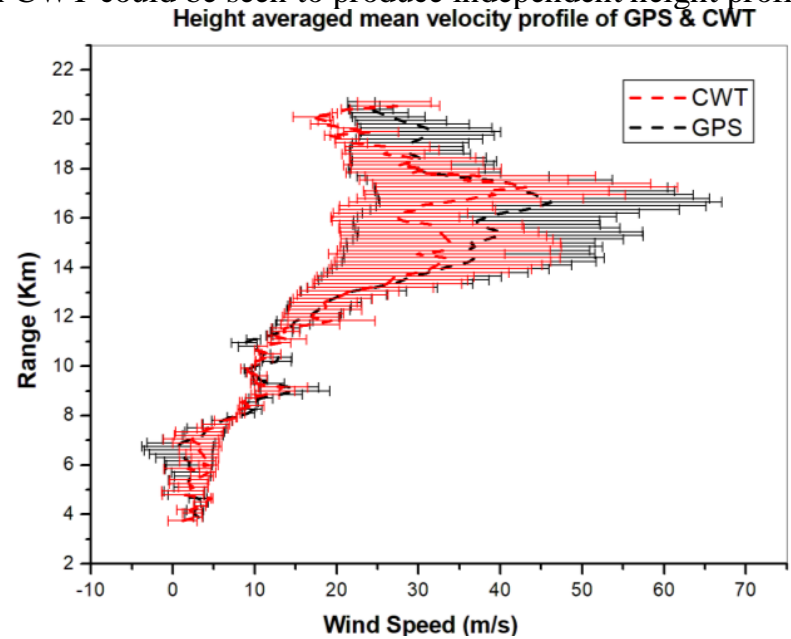

Fig 5.The GPS and the proposed algorithm have a high average mean speed profile

The PALG was able to estimate wind velocities by $\pm 1.8 \mathrm{~m} / \mathrm{s}$ compared to the GPS probe[9], as shown in Fig. 5. It could be attributed again to the fact that in the process of its trajectory the balloon travels horizontally. Figure shows the dispersion of the speed of the wind between the GPS and the PALG. From the figure.6, the high correlation factor of 0.96 indicates that perhaps the wind speed measured by the PAL G is reasonably reliable.

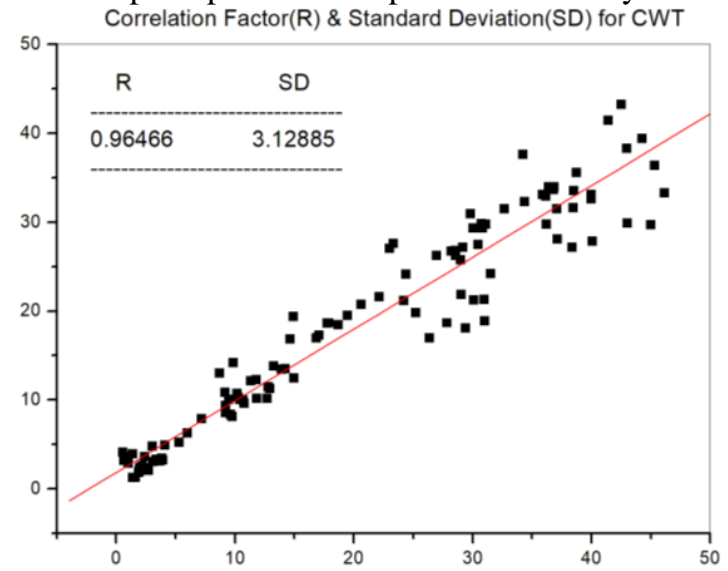

\section{CONCLUSION}

The proposed algorithm exceeds conventional algorithms in all possible respects, Due to the Dual Tree Complex Wavelet Transform shift invariance property, the correlation factor obtainedfrom the existingalgorithm juststays around $85 \%$, whereas it is $96 \%$ for proposed algorithm. Also proposed algorithm can matchup with GPS upto a height of $18 \mathrm{KM}$, whereas existing algorithm fails to achieve it.Its CWT double-tree improves the conventional wavelet transformation which is invariant in practise and directionally selective in higher dimensions. But since 
real and imaginary parts of the dual tree transforms the CWT, the CWT benefits from the detailed theoretical, functional and compute resource creation for the uniform DWT. Software and hardware for implementing the true DWT can, for example, be utilized for CWT directly. However, new effective wavelet-based algorithms can also be exploited in terms of size and phase CWT coefficients, especially in applications with unsuitable or poor DWT performance

\section{REFERENCES}

1. The transformation of the dual-tribe complex wavelet, N.G. Kingsbury, in the proceedings of the 8th IEEE DSP Workshop: new technique for shift inconstance and directional philtres, vol. 8, Bryce Canyon, Utah, USA, August 1998.

2. N. Kingsbury, "The dual-tree complex wavelet transform: a new efficient tool for image restoration and enhancement," in Proceedings of the 9th European Signal Processing Conference (EUSIPCO '98), Rhodes, Greece, September 1998.

3. W. Selesnick, R. G. Baraniuk, and N. G. Kingsbury, "The dual-tree complex wavelet transform," IEEE Signal Processing Magazine, vol. 22, no. 6, pp. 123-151, 2005.

4. W. Y. Wang, H. Z. He, and Z. Y. Zi, "Enhancement of signal denoising and multiple fault signatures detecting in rotating machinery using dual-tree complex wavelet transform," Mechanical Systems and Signal Processing, vol. 24, no. 1, pp. 119-137, 2010.

5. Suresh BabuPotladurty, Dr.G. Sreenivasulu, "Mesosphere Stratosphere Troposphere (MST) Radar Signal processing using Discrete wavelet Transform with Overlapping Group Shrinkage," International Journal of Advanced Science and Technology, vol.28, no. 9, pp.133-136, 2019.

6. D Gabor, 'Theory of Communication', Journal of the IEEE, 93, 429-457, 1946.

7. S. Hahn, 'Hilbert Transforms in Signal Processing', Artech House, Boston, MA, 1996.

8. Adolf Cusmariu, 'Fractional Analytic Signals', Signal Processing, Elsevier, 82, 267 - 272, 2002.

9. V. V. M. JagannadhaRao, D. Narayana Rao, M. VenkatRatnam, K. Mohan, and S. V. B. Rao, "Mean vertical velocities measured by Indian MST radar and comparison with indirectly computed values," $J$. Appl. Meteorol., vol. 42, no. 4, pp. 541-552, Apr. 2003. 